\title{
Postpartale psychische Störungen - Update 2016
}

\author{
Valenka M. Dorsch, Anke Rohde
}

\section{Übersicht}

\author{
Postpartalzeit als Risikofaktor \\ Diagnostische Klassifikation \\ „Babyblues“ (post partum) \\ Postpartale Depression (PPD) \\ Management von \\ Risikopatientinnen
}

$\begin{array}{r}\hline 556 \\ \hline 356 \\ \hline 356 \\ \hline 358 \\ \hline 357 \\ \hline\end{array}$

\section{Differenzialdiagnostik der postpartalen psychischen Störungen} Postpartale psychische Störungen und männliches Geschlecht

\section{Postpartalzeit als Risikofaktor}

In der Zeit nach der Geburt ihres Kindes muss die Mutter eine erhebliche körperliche und insbesondere beim 1. Kind psychische Anpassungsleistung vollbringen. Emotionale Turbulenzen mit psychischer Instabilität sind in dieser Zeit - nicht zuletzt auf dem Hintergrund der ausgeprägten hormonellen Umstellungen nach der Geburt - normal ableitbar und nicht als pathologisches Geschehen anzusehen. Nicht immer ist wegen der „normalen“ Schwierigkeiten mit einer solchen Lebensveränderung gleich zu erkennen, wann sich über den Rahmen der normalen Anpassungsvorgänge hinaus eine psychopathologische Symptomkonstellation entwickelt hat, die eine behandlungsbedürftige postpartale psychische Störung darstellt. Ziel des Beitrags ist es daher, Frauenärzte und Hebammen als die primären Ansprechpartner betroffener Frauen in der Postpartalperiode für charakteristische Störungsbilder und deren Therapiemöglichkeiten zu sensibilisieren und praxisnahe Empfehlungen zur Umsetzung in der gynäkologischen Praxis zu geben. Immerhin stellt die postpartale Depression die zahlenmäßig häufigste medizinische Komplikation im Zusammenhang mit der Geburt eines Kindes in den westlichen Industrieländern dar [1] und betrifft etwa 10-15\% der Mütter nach der Entbindung.

\section{Tipp für die Praxis}

Insgesamt haben Untersuchungen der letzten Jahrzehnte gezeigt, dass keine andere Zeit im Leben von Frauen ein vergleichbar hohes Risiko einer psychischen Erkrankung oder auch Hospitalisierung wegen einer Psychose oder anderen psychischen Störung aufweist wie die Postpartalzeit [2-4].

Darüber hinaus stellt die Geburt eines Kindes, insbesondere auch die des 1 . Kindes eines Paares mit dem Übergang von der Dyade zur Triade und den damit verbundenen Anpassungsleistungen ein relevantes Lebensereignis (Life Event) dar, wie nicht selten im Vorfeld des Auftretens psychischer Störungen zu finden (Vulnerabilitäts-Stress-Modell). In den letzten Jahren hat die Erkenntnis, dass längerfristig bestehende psychische Beschwerden der Mutter erhebliche Auswirkungen auf die emotionale und Verhaltensentwicklung des Kindes haben können [5,6], dazu geführt, dass zunehmend

- spezielle Behandlungskonzepte entwickelt wurden,

- Mutter-Kind-Einheiten in Kliniken eingerichtet worden sind,

- Selbsthilfegruppen und soziale Netzwerke von Betroffenen gegründet wurden.

Das Wissen um postpartale psychische Störungen ist so alt wie die Medizin. Erste Symptombeschreibungen finden sich bei Hippokrates um 400 v. Chr., der in seinem 3. Buch der Epidemien beschrieb, wie eine Frau 
nach der Geburt von Zwillingen ruhelos und verwirrt wurde. Als Ursachen nahm er einen gestörten Lochienfluss, eine Milchstauung oder gar das Eindringen von Muttermilch ins Gehirn an.

\section{Diagnostische Klassifikation}

\section{Postpartale psychische Störungen: keine nosologischen Entitäten}

Eine Reihe von Symptomen einer postpartalen psychischen Störung, insbesondere der postpartalen Depression, ist kaum von physiologischen Anpassungsvorgängen zu unterscheiden, die viele Frauen nach einer Entbindung erleben: Schlafstörungen, Übermüdung, Erschöpfung, verminderte Libido, Veränderungen des Appetits und Stimmungsschwankungen. Gerade das macht oft das Erkennen einer behandlungsbedürftigen „Störung“ so schwierig. Andererseits entsprechen die Symptomkonstellationen einer postpartalen psychischen Störung auch weitestgehend den psychischen Erkrankungen, die unabhängig von Schwangerschaft und Wochenbett auftreten. Einen Überblick über die wichtigsten psychischen Störungen im Zusammenhang mit einer Entbindung gibt Tabelle 1.

\section{Tipp für die Praxis}

Die Begriffe Wochenbettdepression und Wochenbettpsychose sind noch immer sehr geläufig in der Alltagssprache, obwohl inzwischen weitgehend Konsens darüber besteht, dass postpartale psychische Störungen keine nosologische Entität darstellen [7]. Die diagnostische Einordnung erfolgt in den Kategorien für affektive oder psychotische Störungen nach ICD-10 oder DSM-5.

ICD-10. Postpartale Störungen werden nach der jeweils vorherrschenden Symptomatik in den jeweiligen Kategorien für affektive oder psychotische Störungen eingeordnet, also z. B. bei depressiven Episoden in der Kategorie F32 der ICD-10.

Mit dem Zusatz 099.3 zur ICD-10-Codierung F32 kann die Verbindung zum Wochenbett dokumentiert werden.

Nur in Ausnahmefällen - wenn die Symptomatik die Kriterien einer spezifischen Störung nicht erfüllt (in der Regel Subsyndrome, also Symptomkonstellationen, die nicht die vollen Kriterien beispielsweise einer depressiven Episode erfüllen) - kommt die Kategorie F53 zur Anwendung („Psychische oder Verhaltensstörungen im Wochenbett, nicht andernorts klassifiziert“).

DSM-5. Auch im amerikanischen Klassifikationssystem Diagnostic and Statistical Manual of Mental Disorders (DSM-5) werden postpartal auftretende psychische Störungen nach der führenden Psychopathologie in den entsprechenden Kategorien klassifiziert. Für affektive Störungen ist eine Spezifizierung für den peripartalen Beginn vorgesehen, wenn die Störung während der Schwangerschaft oder innerhalb von 4 Wochen nach Entbindung beginnt. Unter dem im englischen Sprachraum verwendeten Begriff Postpartum Depression (PPD) wird in Abgrenzung zum Babyblues einerseits und Postpartum Mood Episode with psychotic Features andererseits üblicherweise eine Major Depression (= depressive Episode) ohne psychotische Symptome mit Beginn in der Schwangerschaft oder bis 4 Wochen post partum verstanden, in verschiedenen Studiendesigns wird der Beginn auch auf das 1. halbe Jahr nach Entbindung erweitert.

\section{„Babyblues“ (post partum)}

Von den behandlungsbedürftigen postpartalen psychischen Störungen abzugrenzen sind die vorübergehenden, nur wenige Stunden bis Tage andauernden Veränderungen in Stimmung und Antrieb, die in den ersten Tagen nach Entbindung etwa die Hälfte aller Frauen betreffen. Die Stimmungsschwankungen bestehen meist in einem Wechsel zwischen Euphorie und Glücklichsein auf der einen und Weinerlichkeit, Reizbarkeit und Insuffizienzgefühlen auf der anderen Seite; wir selbst verwenden deshalb auch den Begriff postpartale affektive Turbulenz [8].

\section{Tipp für die Praxis}

Unter der Vorstellung, dass es sich beim „Babyblues“ um einen physiologisch ableitbaren psychischen Anpassungsvorgang als Folge der abrupten Hormonumstellung nach der Entbindung handelt, ist dafür keine Codierung in ICD-10 oder DSM-5 vorgesehen. Eine spezifische Behandlung ist nicht erforderlich. 
Überblick über die wichtigsten postpartalen psychischen Störungen im Zusammenhang mit einer Entbindung (nach [8]).

\begin{tabular}{|c|c|c|c|}
\hline Störungsbilder & Symptomatik & ICD-10 & Therapie \\
\hline $\begin{array}{l}\text { „Babyblues“ („Heultage“, } \\
\text { postpartale affektive } \\
\text { Turbulenz) }\end{array}$ & $\begin{array}{l}\text { affektive Labilität, erhöhte Emp- } \\
\text { findlichkeit, rascher Wechsel } \\
\text { zwischen Euphorie und Weinen } \\
\text { - Beginn/Dauer: etwa 3.-5. post- } \\
\text { partaler Tag }\end{array}$ & $\begin{array}{l}\text { falls erforderlich O99.3 („Psychische } \\
\text { Störungen und Krankheiten des Nerven- } \\
\text { systems, die Schwangerschaft, Entbin- } \\
\text { dung und Wochenbett komplizieren“) } \\
\text { oder F53 („Psychische oder Verhaltens- } \\
\text { störungen im Wochenbett, nicht andern- } \\
\text { orts klassifiziert“) }\end{array}$ & $\begin{array}{l}\text { - keine spezifische Therapie erforderlich, } \\
\text { empathische Gesprächsführung }\end{array}$ \\
\hline Depression & $\begin{array}{l}\text { - gesamtes Spektrum depressiver } \\
\text { Symptome } \\
\text { - nicht selten Zwangssymptome } \\
\text { und Panikattacken } \\
\text { - verschiedenartige körperliche } \\
\text { Symptome } \\
\text { - lebensmüde Gedanken bis hin } \\
\text { zu manifester Suizidalität } \\
\text { - über mindestens } 2 \text { Wochen } \\
\text { andauernd }\end{array}$ & F32, F33 & $\begin{array}{l}\text { in Abhängigkeit von Ausprägung und Symptom- } \\
\text { konstellation: } \\
\text { — bei leichter Ausprägung supportive Therapie } \\
\text { - ansonsten multimodale antidepressive } \\
\text { Therapie (Medikation, Psychotherapie, } \\
\text { soziale Unterstützung) }\end{array}$ \\
\hline $\begin{array}{l}\text { reaktive Depression } \\
\text { nach perinatalem Verlust } \\
\text { eines Kindes }\end{array}$ & $\begin{array}{l}\text { - in Einzelfällen Entwicklung } \\
\text { pathologischer Trauer bzw. } \\
\text { reaktiver Depression }\end{array}$ & F43 & $\begin{array}{l}\text { - supportive Therapie, Psychotherapie, } \\
\text { ggf. auch Antidepressiva }\end{array}$ \\
\hline $\begin{array}{l}\text { Psychosen/bipolare } \\
\text { Störungen }\end{array}$ & $\begin{array}{l}\text { alle Psychosen kommen vor, } \\
\text { oft manische Symptomatik, } \\
\text { meist akute Psychosen mit } \\
\text { „buntem“ klinischen Bild }\end{array}$ & $F 20, F 23, F 25, F 30, F 31$ & $\begin{array}{l}\text { - dem klinischen Bild entsprechend, Neuro- } \\
\text { leptika, stationäre psychiatrische Behandlung } \\
\text { meist unvermeidbar }\end{array}$ \\
\hline $\begin{array}{l}\text { posttraumatische } \\
\text { Belastungsstörung } \\
\text { nach der Entbindung }\end{array}$ & $\begin{array}{l}\text { - Geburtssituation wurde trauma- } \\
\text { tisch erlebt. Wiedererleben in } \\
\text { Intrusionen, „Flashbacks“, } \\
\text { Alpträumen. Folge nicht selten } \\
\text { Vermeidungsverhalten, sozialer } \\
\text { Rückzug, Gefühl innerer Stumpf- } \\
\text { heit, Gereiztheit, erhöhte } \\
\text { Schreckhaftigkeit } \\
\text { - auch Reaktualisierung früherer } \\
\text { traumatischer Erfahrungen mög- } \\
\text { lich (z. B. sexueller Missbrauch) }\end{array}$ & $\begin{array}{l}\text { F43. } 1 \\
\text { (umstritten, ob „Geburt als } \\
\text { Trauma“ die Kriterien erfüllt) } \\
\text { sonst andere F43-Kategorie }\end{array}$ & $\begin{array}{l}\text { - Psychotherapie, möglichst traumaspezifisch } \\
\text { - bei depressiver Begleitsymptomatik } \\
\text { ggf. antidepressive Behandlung (für PTBS } \\
\text { zugelassene Antidepressiva) }\end{array}$ \\
\hline Angststörungen & $\begin{array}{l}\text { - oft Panikstörung, manchmal auch } \\
\text { Exazerbation vorbestehender } \\
\text { generalisierter Angststörung } \\
\text { - Panikattacken können auch Teil } \\
\text { postpartaler Depression sein }\end{array}$ & dann F32, F33 & $\begin{array}{l}\text { nach den üblichen Regeln der Therapie bei } \\
\text { Angststörungen (Psychotherapie und/oder } \\
\text { Antidepressiva) } \\
\text { - Behandlung der postpartalen Depression }\end{array}$ \\
\hline Zwangserkrankungen & $\begin{array}{l}\text { - Zwangssymptome (i.d. R. mit } \\
\text { dem Inhalt, dem eigenen Kind } \\
\text { etwas anzutun) } \\
\text { - Zwangssymptome können auch } \\
\text { Teil postpartaler Depression sein }\end{array}$ & $\begin{array}{l}\text { F42 } \\
\text { dann F32, F33 }\end{array}$ & - Psychotherapie, ggf. auch Antidepressiva \\
\hline
\end{tabular}




\section{Physiologische Anpassungsvorgänge}

Epidemiologie und Ätiopathogenese. Etwa 50-70\% aller Frauen sind um den 3.-5. Tag post partum vom „Babyblues“ betroffen [7], was einen ersten Hinweis darauf gibt, dass es sich dabei nicht um eine pathologische Veränderung im engeren psychiatrischen Sinne handelt. Es ist vielmehr davon auszugehen, dass die abrupte physiologische Hormonumstellung in den ersten Tagen nach der Entbindung dafür (mit-)verantwortlich ist. Während der Schwangerschaft kommt es durch die Hormonbildung der Plazenta zu bis zu 200fachen Werten für Östrogene und Progesteron, in den ersten 3-5 Tagen nach Entbindung erfolgt dann ein sehr rascher Abfall auf normale bzw. subnormale Werte.

Selbstlimitierender Verlauf. Eine spezifische Behandlung ist beim „Babyblues“ in der Regel nicht erforderlich, spätestens nach 2-3 Tagen klingt die Symptomatik von selbst ab. Ein empathischer und sensibler Umgang mit der Patientin und unterstützende Gespräche z.B. mit der Hebamme werden von den betroffenen Frauen als hilfreich erlebt. Nur selten kommt es zum Übergang in eine postpartale Depression oder Psychose.

Ausgeprägte und schwere Symptome in der Phase des „Babyblues“ gelten als Vulnerabilitätsmerkmal für das Auftreten einer postpartalen Depression [9].

\section{Tipp für die Praxis}

Die Diagnose einer Anpassungsstörung (ICD-10: F43.2) sollte nur vergeben werden, wenn es tatsächlich konkrete äußere Ereignisse gibt, an denen sich die Symptomatik festmacht (wie etwa eine reaktive Depression nach dem perinatalen Verlust eines Kindes oder nach einer Frühgeburt). Diese Fälle werden in der Regel psychotherapeutisch behandelt.

\section{Postpartale Depression (PPD)}

\section{Häufigste Störung in der Postpartalzeit}

Epidemiologie. Depressive Symptome sind bezüglich Häufigkeit und Folgen die wichtigsten psychopathologischen Symptome post partum. In den meisten Studien entsprechen die Prävalenzraten peripartaler Depressionen denen von Frauen in anderen Lebenssituationen (antenatal: 10-12\%; postnatal: 10-20\%; $[10,11])$. Eine Metaanalyse über 149 Studien aus
40 Ländern zeigte eine Variabilität von 0,5-60\%, mit höheren Prävalenzen in Ländern mit niedrigem bis mittlerem Einkommen [12]. Nicht immer ist postpartale Depressivität jedoch gleichbedeutend mit dem Vollbild einer depressiven Episode, insbesondere, wenn mittels der „Edinburgh Postnatal Depression Scale“ (EPDS) erhoben. Aber auch wenn man von niedrigen Zahlen ausgeht und eine klinisch relevante postpartale depressive Symptomatik bei nur 10\% der Frauen annimmt, bedeutet dies bei etwa 710000 Geburten pro Jahr in Deutschland, dass über 70000 Frauen pro Jahr unter der Symptomatik einer postpartalen Depression leiden und mit ihnen ihre Kinder und Partner.

Postpartale Depression ist in Ländern mit hohem ökonomischem Status (High Income Countries) die häufigste Todesursache von Müttern in der Perinatalzeit.

Ätiopathogenese. In den letzten Jahren konnte eine Zunahme bei der Diagnose von depressiven Erkrankungen verzeichnet werden. Die postpartalen Depressionen sind dabei ein gutes Beispiel, welchen Einfluss kulturelle und gesellschaftliche Bedingungen im Sinne einer multifaktoriellen Genese der Erkrankung darstellen. Bei einer zu postulierenden Vulnerabilität zur Entwicklung von Depressionen tragen hierbei insbesondere die Veränderungen im familiären und sozialen Umfeld mit Reduktion auf Kleinfamilien, spezielle Vorbereitungen und Planung der wenigen Schwangerschaften im Leben von Frauen sowie hohe eigene Ansprüche an ein besonderes Mutterbild bei - nicht zuletzt vermittelt durch die Darstellung in den Medien. Als Risikofaktoren für das Auftreten postpartaler Depressionen konnten zahlreiche biologische und psychosoziale Faktoren identifiziert werden [9-12], darunter u.a.

- geringe soziale Unterstützung

- konfliktreiche Partnerschaft

- häusliche Gewalt

- Missbrauchserfahrung

- Migration

- Vorgeschichte mit affektiven Störungen, Angststörungen oder prämenstruellem Syndrom

- ausgeprägter Babyblues nach der Entbindung

- Depressionen in der Schwangerschaft

- Substanzmissbrauch

- Frühgeburt

- maternale chronische Erkrankung

- endokrine Faktoren

- eine Persönlichkeit mit hohem Selbstanspruch 
Bisher untersuchte Strategien zur pränatalen Prävention zeigen kleine Effekte psychosozialer Interventionen (Hausbesuche, Telefonsupport) und im Übrigen uneinheitliche Ergebnisse [13]. Ein gewisser protektiver Effekt wird dem Grad sozialer Unterstützung, insbesondere durch den Partner, zugeschrieben.

\section{Fazit für die Praxis}

Auch wenn eine Reihe von Risikofaktoren benannt werden kann, zeigen bisher untersuchte Strategien zur pränatalen Prävention uneinheitliche Ergebnisse. Insbesondere in der Praxis kann aus dem Vorhandensein oder Fehlen von Risikofaktoren nicht zwanglos abgeleitet werden, ob eine Frau tatsächlich betroffen sein wird.

Pathophysiologie. Gegenstand der wissenschaftlichen Diskussion um die pathophysiologischen Grundlagen der postpartalen Depression ist seit einigen Jahren der Zusammenhang zwischen postpartalen affektiven Symptomen und endokrinen Faktoren, insbesondere den ausgeprägten Veränderungen gonadaler Steroide während der Schwangerschaft und in der Postpartalzeit. Insgesamt konnten in Untersuchungen keine konstant nachweisbaren Unterschiede in den Spiegeln gonadaler Steroide in Schwangerschaft oder Wochenbett bei Frauen mit bzw. ohne postpartaler Depression belegt werden, sodass weder der reine Steroidanstieg noch der -entzug post partum für die Entwicklung der PPD verantwortlich gemacht werden kann. Die Ergebnisse legen jedoch nahe, dass bei den betroffenen Frauen eine individuell vorhandene erhöhte Sensitivität für die stimmungsverändernden Effekte gonadaler Steroide angenommen werden muss, ähnlich wie es auch für die prämenstruelle dysphorische Störung postuliert wird [9]. Andere neurobiologische Theorien postulieren darüber hinaus auch noch ZNS-Veränderungen im Sinne modifizierter Zytokine und Fettsäuren, eine Imbalance der Hormone auf der HPA-Achse sowie veränderte Oxytocin- und Vasopressinspiegel als konstellierende Faktoren [14].

\section{Symptomatik postpartaler Depressionen}

Postpartale Depressionen zeigen das gesamte Spektrum depressiver Symptomatik mit gedrückter Stimmung, Antriebsverminderung und Interessenverlust, verminderter Genuss- und Freudfähigkeit, Angstsymptomen, Grübeln mit Auftreten von Suizidgedanken sowie psychomotorische und kognitive Beeinträchtigungen, wie sie zu jedem anderen Zeitpunkt im Leben im
Rahmen einer depressiven Episode auftreten können. Psychotische Symptome sollten dabei fehlen.

\section{Tipp für die Praxis}

Das klinische Bild einer postpartalen Depression ist von einer depressiven Episode in anderen Lebensabschnitten nicht zu unterscheiden, allerdings kommen thematisch Symptome mit Bezug zur Mutterschaft (z. B. Insuffizienz- und Schuldgefühle als Mutter) hinzu.

Gestörte Mutter-Kind-Interaktion. Typisch für die postpartale Depression sind erhebliche Zweifel der betroffenen Frau an ihrer Qualität als Mutter - üblicherweise abgeleitet aus dem Gefühl, keine „richtigen“ Muttergefühle gegenüber dem Neugeborenen empfinden oder das Baby nicht lieben zu können (zu interpretieren als das für eine Depression typische Symptom Gefühl der Gefühllosigkeit).

Depressive Symptome nach einer Entbindung entwickeln sich typischerweise schleichend während der ersten Wochen, können sich aber auch erst innerhalb des 1. postpartalen Jahres ausprägen [15].

Besonders schlimm ist es für betroffene Mütter, wenn im Rahmen der postpartalen Depression Zwangsgedanken hinzukommen, also sich immer wieder ungewollt aufdrängende Gedanken - meist mit dem Inhalt, dem eigenen Kind etwas anzutun. Darüber hinaus sind aber auch ein mangelndes Verständnis für die Bedürfnisse des Säuglings, Passivität, weniger positiver Affekt und reduziertes mimisches Ausdrucksverhalten sowie ein Mangel an Empathie und emotionaler Verfügbarkeit gegenüber dem Kind als Ausdruck einer gestörten Mutter-Kind-Interaktion charakteristisch. Insbesondere diese Symptome machen die Notwendigkeit einer raschen Behandlung deutlich, um nachhaltige Störungen der Mutter-Kind-Bindung zu vermeiden [15], zumal ja Studien der letzten Jahre solche Auswirkungen belegen (s.o.).

\section{Tipp für die Praxis}

Entsprechen die Symptome einem depressiven Syndrom mit einer Dauer von mindestens 2 Wochen, kann die Diagnose einer depressiven Episode mit postpartalem Beginn gestellt werden. Der Ausprägungsgrad (leicht, mittelgradig oder schwer) entspricht den ICD-10-Diagnosekriterien. 


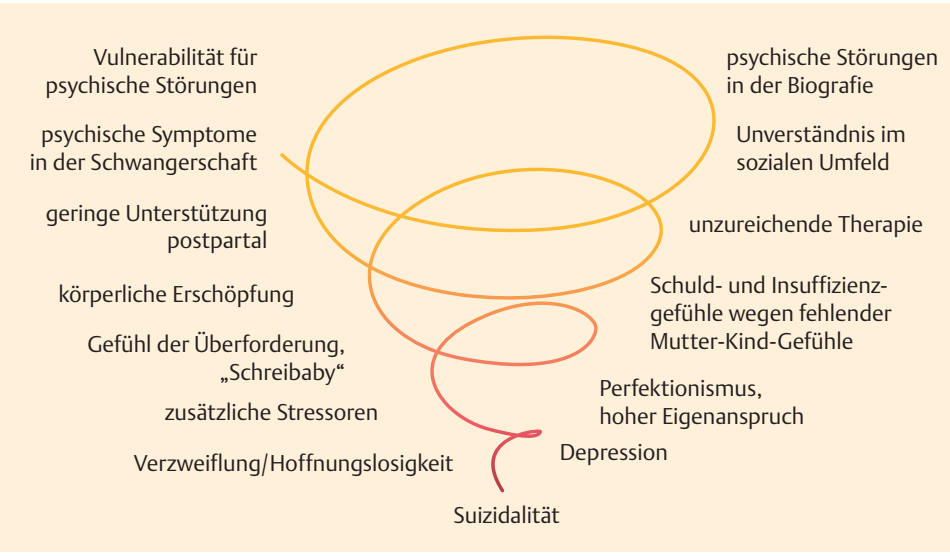

Abb. 1 Depressive Spirale der postpartalen Depression (aus: [16]). wenn die Mutter ihr Kind nicht alleine lassen will und es tötet, bevor sie selbst den Suizidversuch macht. Abb. 1 zeigt die depressive Spirale der postpartalen Depression.

\section{Tipp für die Praxis}

Als wichtiger Hinweis auf eine dringend behandlungsbedürftige postpartale Depression sind solche schweren Insuffizienzgefühle zu werten; wenn ausgeprägte Hoffnungslosigkeit hinzukommt, muss die Suizidgefahr besonders genau überprüft werden. Diese Form der postpartalen Depression führt leicht zur Chronifizierung und auch zu einer Zuspitzung der Symptomatik bis hin zu Suizidalität (depressive Spirale der postpartalen Depression).

In der Regel ist eine antidepressive Medikation erforderlich, meist auch gut wirksam.

Da häufig Schlafstörungen im Vordergrund stehen, bietet sich die Gabe eines schlafanstoßenden und anxiolytischen Antidepressivums an. Wie im Fallbeispiel 1 muss nach sorgfältiger Nutzen-Risiko-Abwägung der betroffenen Frau auch bei medikamentöser Therapie nicht zwingend zum Abstillen geraten werden, da dies oft von den Frauen als zusätzliches „Versagen“ empfunden wird (s.a. [18]).

\section{Zwangstyp}

gefühle im Vordergrund stehen und von einer Vielzahl anderer depressiver Symptome begleitet werden. Ausgehend von zunächst „ganz normalen Unsicherheiten“ verstärken sich die Ängste betroffener Frauen und weiten sich zunehmend zu der Überzeugung aus, eine schlechte Mutter zu sein. Dieses Symptom, das psychopathologisch als Gefühl der Gefühllosigkeit einzuordnen ist, ist oft verbunden mit der Wahrnehmung, dass die eigenen Muttergefühle nicht den Erwartungen entsprechen. Selbst Frauen, die bewusst schwanger geworden sind und sich mit viel Freude auf die Mutterschaft vorbereitet haben, wissen plötzlich nicht mehr, warum sie eigentlich ein Kind wollten bzw. wie sie sich je zugetraut haben, dies bewältigen zu können. Die Ambivalenz kann soweit führen, dass betroffene Mütter überlegen, ob es nicht besser wäre, das Kind „zurückgeben zu können“ oder es zur Adoption freizugeben. Nicht selten resultieren diese Überlegungen auch in Suizidalität („Wenn ich mir das Leben nehme, kann mein Mann neu heiraten, und dann bekommt mein Kind wenigstens eine richtige Mutter.“). Darüber hinaus besteht auch die Gefahr des erweiterten Suizids,
Mit ca. 25\% weniger häufig, aber für die betroffenen Frauen sehr quälend, sind postpartale Depressionen, bei denen Zwangssymptome im Vordergrund stehen. In der Regel handelt es sich um den sich immer wieder aufdrängenden Gedanken, dem eigenen Kind etwas anzutun (= Obsession of Infanticide [19]), z. B. den Kinderwagen an einer abschüssigen Stelle loszulassen, das Kind vom Arm fallen zu lassen, es zu erstechen, zu ersticken, im Badewasser zu ertränken. Diese Gedanken verursachen bei den betroffenen Frauen große Ängste. Die Folge sind ausgeprägte Scham- und Schuldgefühle. Bei vielen dieser Frauen findet sich bereits ein deutliches Vermeidungsverhalten, z. B. das Kind nicht mehr zu baden, wenn sie mit ihm alleine sind, oder alle scharfen Messer aus der Küche zu verbannen.

Entlastend ist für Frauen in dieser Situation die Aufklärung über die Art der Symptome und den Charakter von Zwangsgedanken, dass diese nämlich nicht umgesetzt werden. Oft sind schon die Thematisierung und die Information darüber, dass gar nicht so selten Mütter 
Fallbeispiel 1: Insuffizienztyp [16]

\section{Am liebsten hätte ich das Kind zurückgegeben}

Die 34-jährige Frau M. stellt sich 4 Monate nach der Entbindung ihres 1. Kindes in der Gynäkologischen Psychosomatik am Zentrum für Frauenheilkunde und Geburtshilfe des Universitätsklinikums Bonn vor. Ihre Tochter - ein Wunschkind - entwickelt sich gut und ist ein „pflegeleichtes“ Kind. Gerade deshalb hat Frau M. ausgeprägte Schuldgefühle, weil sie dem Kind gegenüber noch immer keine Muttergefühle entwickelt habe. Sie macht sich Vorwürfe, ist niedergeschlagen, kann kaum essen oder schlafen - auch wenn das Kind selbst ruhig schläft - und hat kaum Antrieb. Frau M. selbst hat für alles eine Erklärung („Nachts kann ich nicht schlafen, weil ich ja das Kind hören muss; morgens komme ich nicht aus dem Bett, weil ich noch müde bin..."), was ihr das Erkennen der Depression sehr schwer macht. Ihr kommt es vor, als hätten andere Mütter diese Probleme nicht („Die haben alles im Griff, nur ich nicht."). Ihr kommt immer häufiger der Gedanke, dass es besser wäre, wenn sie das Kind „zurückgeben“ könnte. Auch erste lebensmüde Gedanken sind aufgetaucht, die sie aber noch jederzeit zurückdrängen kann. In der Diagnostik ergeben sich Hinweise auf eine postpartale
Depression vom Ausmaß einer mittelschweren Episode, wobei Insuffizienz- und Schuldgefühle zentrale Symptome sind. Da Frau M. gerne weiterstillen möchte („Stillen ist doch das einzig Gute, was ich für mein Kind noch tun kann. ") wird nach einer NutzenRisiko-Abwägung eine antidepressive Medikation eingeleitet. Bereits mit einer niedrigen Tagesdosis verbessert sich ihr Schlaf, die depressive Symptomatik klingt rasch ab. Bald entwickelt Frau M. eine intensive Beziehung zu ihrer Tochter. Die Empfehlung, wegen der deutlich werdenden primären Selbstwertproblematik eine Psychotherapie anzuschließen, nimmt sie auf.

\section{Fallbeispiel 2: Zwangstyp [16]}

\section{Sieht so eine Mörderin aus?}

Diese Frage stellt sich Frau X., 28 Jahre alt, immer wieder, wenn sie sich im Spiegel ansieht. Etwa 5 Wochen nach der Geburt ihres erwünschten 2 . Kindes hat sie zunehmend häufiger den Gedanken, ihrem Kind etwas anzutun. Wenn ihr Sohn badet, sieht sie ihn plötzlich unter Wasser liegen; wenn sie am Fenster steht, stellt sie sich vor, wie er hinunterfällt. Es hat sich bereits ein ausgeprägtes Vermeidungsverhalten entwickelt: Sie hat alle Messer versteckt, badet das Kind nur noch, wenn ihr Mann zu
Hause ist. Sie schämt sich schrecklich für die Gedanken, kann sich nicht vorstellen, dass es andere Mütter gibt, die solche Gedanken haben. Frau X. entwickelt eine zunehmend depressive Symptomatik, wegen der sie sich in der Gynäkologischen Psychosomatik vorstellt. Bei der Exploration fragt die Psychiaterin auch ganz gezielt nach solchen Gedanken („Es gibt Mütter, die haben nach der Entbindung die Angst, dass sie ihrem Kind etwas antun könnten; kennen Sie das auch?"). Dadurch ermutigt, spricht die junge Mutter das 1. Mal mit einem anderen Menschen über ihre Gedanken. Die Information erleichtert sie, dass es sich bei Zwangsgedanken um kein seltenes Phänomen handelt, von dem viele Frauen nach der Entbindung betroffen sind. Ganz besonders entlastet wird Frau X. durch die Aufklärung, dass Zwangsgedanken zwar unangenehm sind, aber nicht in die Praxis umgesetzt werden. Strategien zum Umgang mit Zwangsgedanken kann Frau X. rasch erlernen und diese auch gut umsetzen, sodass im Verlauf eine Medikation nicht erforderlich ist. nach der Entbindung solche Symptome erleben, hilfreich. Auch die Ermutigung, sich mit anderen Betroffenen, z.B. über eine Selbsthilfegruppe (www.schattenund-licht.de), auszutauschen, entlastet die Frauen sehr. Therapeutisch steht die Behandlung der postpartalen Depression nach einer multimodalen Therapiestrategie im Vordergrund, wobei die Patientin zusätzlich verhaltenstherapeutisch den Umgang mit Zwangsgedanken erlernt.

\section{Tipp für die Praxis}

Für den behandelnden Therapeuten ist die sichere Abgrenzung entscheidend, dass es sich um Zwangsgedanken handelt und nicht etwa um akustische Halluzinationen mit imperativem Charakter, um das Risiko für die Betroffene und insbesondere auch für ihr Kind einschätzen zu können (Tab. 2). 
Tabelle 2

Gefährdungsmomente für das Kind bei postpartalen psychischen Störungen (nach [16]).

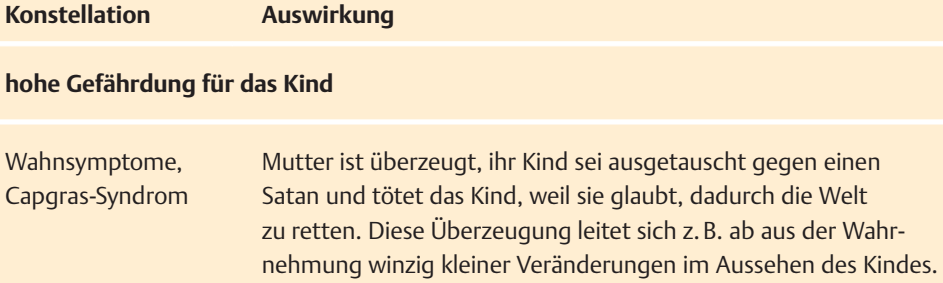

Wahnsymptome, Capgras-Syndrom

Mutter ist überzeugt, ihr Kind sei ausgetauscht gegen einen Satan und tötet das Kind, weil sie glaubt, dadurch die Welt zu retten. Diese Überzeugung leitet sich z. B. ab aus der Wahrnehmung winzig kleiner Veränderungen im Aussehen des Kindes.

akustische Halluzinationen, imperative Stimmen (Stimmen mit Befehlscharakter)

erweiterter Suizid

Neonatizid

Zwangsgedanken
Stimmen, die die Mutter auffordern, bestimmte Handlungen vorzunehmen (z. B. „Wirf das Kind aus dem Fenster, es ist das Kind böser Eltern. “) werden oft umgesetzt und stellen eine hohe Gefährdung dar.

Mutter ist depressiv, hoffnungslos und suizidal; möchte ihr Kind nicht alleine auf der Welt zurücklassen, tötet es zuerst, dann sich selbst.

Tötung des Neugeborenen direkt nach der Geburt, i.d. R. nach verdrängter oder verleugneter Schwangerschaft; aktive Tötung (z. B. um das Kind am Schreien zu hindern) oder passive Tötung (durch unterlassene Versorgung, Unterkühlung etc.)

\section{keine Gefährdung für das Kind}

Gedanken mit fremdaggressivem Charakter (z. B. das Kind mit einem Messer zu verletzen), die sich unwillkürlich aufdrängen. Die Mutter ist selbst entsetzt, weil sie weiß, dass sie so etwas nie tun würde. Trotzdem starke Schuld- und Schamgefühle und erheblicher Leidensdruck.

\section{Paniktyp}

Eher selten, dann aber für die Betroffene und ihre Familie sehr belastend, kann die postpartale Depression von häufigen und ausgeprägten Panikattacken begleitet sein. Auch Frauen, die zuvor nie unter einer Angstoder Panikstörung gelitten haben, können postpartal davon betroffen sein. Anders als bei der differenzialdiagnostisch davon abzugrenzenden Panikstörung (ICD-10: F41.0), bei der die Panikattacken das zentrale Symptom sind und die depressive Symptomatik eine Begleiterscheinung darstellt, sind die Panikattacken bei diesem Typ der postpartalen Depression Teil der ausgeprägten depressiven Symptomatik, die mit einer Besserung der Depression auch wieder abklingen. Im Rahmen eines multimodalen Therapieregimes aus Medikation, Psychotherapie und sozialer Unterstützung bieten sich hier insbesondere Antidepressiva vom SSRI-Typ an.
Benzodiazepine wie Lorazepam oder Alprazolam, die zwar gut angstlösend sind, aber auch ein hohes Abhängigkeitspotenzial haben, sollten möglichst vermieden werden.

\section{Diagnostik und Differenzialdiagnose}

Nicht immer ist die Abgrenzung einer postpartalen Depression von dem, was zu den „normalen“ Reaktionen nach einer Entbindung zählt, für Patientinnen und ihre Angehörigen einfach. Dies gilt auch für die die jungen Familien in dieser Zeit betreuenden Nachsorgehebammen, Haus- und Frauenärzte. Zur Diagnostik postpartaler Depressionen gehört zunächst immer der Ausschluss organischer Ursachen (z. B. Anämie, Schilddrüsenfunktionsstörung). Weitere endokrine Imbalancen, z. B. ein ausgeprägter Östrogenmangel nach der Entbindung, werden nach aktuellem wissenschaftlichen Stand am ehesten als konstellierende Faktoren der postpartalen Depression diskutiert.

EPDS - Edinburgh Postnatal Depression Scale. Beim Screening auf postpartale Ängste und Depressivität empfiehlt sich die auch in einer deutschen Fassung vorliegende Edinburgh Postnatal Depression Scale, EPDS, die eine leichte Identifizierung von betroffenen Müttern und Vätern in der Schwangerschaft und Postpartalzeit ermöglicht [20,21]. Der 10 Fragen umfassende Selbstbeurteilungsfragebogen kann Werte zwischen 0 und 30 annehmen, der Cutoff liegt bei einem EPDS-Score $>12$, wonach eine weiterführende fachpsychiatrische Diagnostik erfolgen sollte.

EPDS-Werte über 20 können nach der eigenen klinischen Erfahrung schon fast als Beleg einer akuten depressiven Störung gewertet werden.

Die Sensitivität und Spezifität der EPDS hat sich in den vergangenen 2 Jahrzehnten als sehr gut erwiesen, sodass der Einsatz im klinischen Alltag, aber auch im Rahmen von Forschungsprojekten empfohlen werden kann $[1,22]$. Inzwischen ist die EPDS auch ein gut akzeptiertes Screening-Instrument in der gynäkologischen Praxis, weshalb der gezielte Einsatz der EPDS in den niedergelassenen Frauenarztpraxen zum Zeitpunkt der Nachuntersuchung nach der Entbindung (ca. 6 Wochen post partum) empfohlen wird, um bereits betroffene oder Frauen mit Risiko für eine depressive Entwicklung zu erkennen. Dadurch könnte eine zügige Diagnosestellung der noch immer unter- bzw. zu spät diagnostizierten postpartalen Depressionen in Deutschland erzielt werden. Da ein Großteil der post- 
partalen Depressionen bereits in der Schwangerschaft beginnt, ist auch der präpartale Einsatz der EPDS zur Identifizierung von Risikopatientinnen vielversprechend [22].

Screening-Fragen nach Whooley. Ebenso validiert wie die EPDS, aber noch niederschwelliger, z. B. bei fremdsprachigen Patientinnen, können die sog. ScreeningFragen (nach Whooley) [23] einen hilfreichen Gesprächseinstieg in der gynäkologischen oder Hebammenpraxis bieten.

\section{Verlauf und Prognose}

Insbesondere wenn es sich um die Erstmanifestation einer depressiven Störung nach der Entbindung handelt, ist diese in der Regel gut behandelbar, meist ist ein rascher Therapieeffekt zu erzielen - unabhängig davon, ob rein psychotherapeutische oder medikamentöse Strategien oder eine Kombination von beidem eingesetzt werden. Eine möglichst frühe und effiziente Behandlung ist nicht nur für das subjektive Befinden der Mutter und die Stabilität der Familie von Bedeutung, sondern auch für die Entwicklung des Kindes. Lang andauernde bzw. chronifizierte Depressionen können über eine Störung der Mutter-Kind-Bindung zu negativen Einflüssen in der emotionalen und kognitiven kindlichen Entwicklung führen [15]. In Studien an 600 Mutter-Kind-Paaren konnten schon im 1. Lebensmonat Veränderungen im Verhalten der Kinder bei von PPD betroffenen Müttern im Vergleich zu Kindern von gesunden Müttern gefunden werden [24].

\section{Postpartale Depressionen und Suizidalität}

Nach Studienlage sind die Raten an Suizidversuchen bei Frauen in der Schwangerschaft und postpartal niedriger als zu anderen Zeiten im Leben $[1,24]$. Gleichzeitig sind die gewählten Methoden bei den in dieser Zeit dokumentierten Suiziden gewaltsamer und enden häufiger tödlich. Als Risikofaktoren für Suizidalität in der Postpartalzeit konnten vorangegangene Suizidversuche, Hoffnungslosigkeit, Substanzmissbrauch und Gewalterlebnisse in der Partnerschaft benannt werden und sollten daher Gegenstand sorgfältiger Exploration bei von PPD betroffenen Frauen sein.
Einfaches Screening in der gynäko.

logischen Praxis mittels EPDS

Der in einer deutschen Fassung vorliegende Edinburgh Postnatal Depression Scale, EPDS, ein von der Patientin in wenigen Minuten auszufüllender und sehr einfach auszuwertender Selbstbeurteilungsfragebogen (abrufbar unter www. schatten-und-licht.de) ist ein geeignetes Instrument, um Mütter beim 1. Praxisbesuch nach der Entbindung auf erhöhte Depressivität und/oder Angst zu untersuchen. Idealer Zeitpunkt für dieses Screening ist der 1. Vorstellungstermin nach der Entbindung in der Frauenarztpraxis; schon im Wartezimmer kann die Patientin den Bogen ausfüllen und dieser liegt dann beim ärztlichen Kontakt bereits vor. Bei Bedarf kann dann näher auf die Frage des psychischen Befindens eingegangen werden, um eine erste Beurteilung der Behandlungsbedürftigkeit vornehmen zu können.

\section{Screening-Fragen (nach Whooley)}

- „Fühlten Sie sich im letzten Monat häufiger niedergeschlagen, traurig, bedrückt oder hoffnungslos?“

- „Hatten Sie im letzten Monat deutlich weniger Lust und Freude an Dingen, die Sie sonst gerne tun?"

Falls beide Fragen mit „Ja“ beantwortet werden, sollte eine weiterführende Diagnostik erfolgen. Ideale Zeitpunkte für die Screeningfragen:

- beim 1. Kontakt und

- 4-6 Wochen postpartal.

\section{Tipp für die Praxis}

Es ist wichtig, an die Gefahr durch erweiterten Suizid zu denken, bei der die Frau zunächst das Kind, dann sich selbst tötet (s. a. Tab. 2). Auch die frühzeitige Erkennung etwaiger psychotischer Symptome (die dann differenzialdiagnostisch für eine postpartale Psychose sprechen) ist ganz entscheidend, um Gefährdungsmomente für Mutter und Kind zu erkennen. Wann immer diesbezüglich Unsicherheit besteht, muss die Verweisung an einen Psychiater oder besser noch eine psychiatrische Notfallambulanz erfolgen.

Rezidivrisiko. Im Hinblick auf weitere Schwangerschaften und Geburten haben die betroffenen Mütter oft große Sorge, wieder krank zu werden. Auch wenn sie insgesamt ein erhöhtes Risiko einer postpartalen Depression nach einer weiteren Geburt haben, kann man sie diesbezüglich entlasten, da die „psychologischen Belastungen" einer 1. Geburt und Mutterschaft entfallen. Die Frauen sollten aufgeklärt und ermutigt werden, sich im Falle einer erneuten Schwangerschaft schon vor der Geburt in ambulanter psychiatrischer Behandlung vorzustellen und v.a. einen „Vorsorge- 
termin“ ca. 3-4 Wochen nach der Geburt zu vereinbaren (dann auch Nutzung der EPDS als ScreeningInstrument).

Bei einer erneuten Schwangerschaft besteht zwar ein erhöhtes Risiko einer weiteren postpartalen Depression, dem kann aber durch Vorsorge rechtzeitig gegengesteuert werden.

Aus unserer klinischen Erfahrung hat es sich als hilfreich erwiesen, mit den Frauen bereits frühzeitig das Management von Schwangerschaft, Entbindung und Postpartalzeit zu besprechen, um die oft erheblichen Ängste der Frauen, mit einer erneuten Schwangerschaft auch eine erneute Episode zu erleiden, abbauen zu können. Auch über die gezielte Nutzung aller Ressourcen im sozialen Umfeld (Unterstützung durch den Partner z. B. durch Elternzeit/Vätermonate, Familie und Freunde) oder durch Programme wie „Frühe Hilfen“ (z.B. Familienhebamme) sowie Entlastung durch Beantragung einer Haushaltshilfe bei der Krankenkasse sollte dabei gesprochen werden.

\section{Tipp für die Praxis}

Gerade im Fall ungeplant eingetretener Schwangerschaften erwägen Frauen - aus Angst vor einer Wiedererkrankung - nach einer postpartalen Depression manchmal einen Schwangerschaftsabbruch, obwohl das Kind eigentlich erwünscht ist.

In manchen Fällen ist nach fundierter Nutzen-RisikoAbwägung eine medikamentöse Prophylaxe schon während der Schwangerschaft oder zumindest unmittelbar nach der Entbindung sinnvoll, insbesondere wenn es Hinweise auf eine bipolare Störung oder psychotische Symptome in der Anamnese gibt. Frauen mit einer solchen Vorgeschichte sollten die Gestaltung der Peripartalzeit mit Geburtsvorbereitung, Stressreduktion, Planung einer eventuell erforderlichen Medikation und des Stillens, Möglichkeiten der sozialen Unterstützung etc. frühzeitig mit ihrem Psychiater besprechen, wobei auch der Partner oder andere Familienangehörige einbezogen werden sollten (weiterführende Informationen zum peripartalen Management s. [18]).

\section{Leitliniengerechte Therapie}

\section{Multimodaler Therapieansatz}

Die Therapie einer postpartalen Depression erfolgt analog den Leitlinien einer antidepressiven Behandlung. In der 2015 neu aufgelegten S3-Leitlinie „Unipolare Depression“ sind peripartale Depressionen als eigenständige Kategorie aufgeführt [25]. Die Therapie ist in der Regel multimodal, sowohl medikamentös als auch psychotherapeutisch und soziotherapeutisch (soziale Unterstützung). Neben der Schwere der Episode und Berücksichtigung der im Vordergrund stehenden Symptomatik spielt auch die Frage, ob die betroffene Mutter stillt bzw. weiter stillen möchte, eine wegweisende Rolle bei der Therapieentscheidung.

Bei schweren postpartalen Depressionen ist eine psychopharmakologische Behandlung in der Regel unumgänglich.

Bei stillenden Müttern ist mit der Entscheidung für Psychopharmaka eine fundierte Nutzen-Risiko-Abwägung und die sorgfältige Wahl des Präparats erforderlich (s.a. [18]).

\section{Tipp für die Praxis}

Bei den meisten Antidepressiva ist bei Monotherapie unter Nutzen-Risiko-Abwägung das Stillen möglich [18]. Der Wunsch der Mutter nach der Weiterführung des Stillens schließt eine antidepressive Medikation vom Grundsatz her nicht aus.

Die Wahl des Antidepressivums folgt dabei der Zielsymptomatik: Sind Erschöpfung und Antriebsminderung im Rahmen des depressiven Syndroms für die Mutter besonders belastend, empfiehlt sich eine Substanz aus der Klasse der antriebssteigernden SSRI/ SSNRI (z.B. Sertralin, Citalopram, Venlafaxin). Nicht selten aber stehen Agitiertheit, Unruhe und ausgeprägte Schlafstörungen im Vordergrund, sodass eher eine schlafanstoßende/sedierende Substanz (z.B. Mirtazapin oder Amitriptylin) infrage kommt.

Bei Patientinnen mit einer antidepressiven Behandlung in der Vorgeschichte spricht in der Regel auch nichts dagegen, das Medikament, mit dem sie zuvor gute Erfahrungen gemacht hat, auch bei der postpartalen Manifestation der depressiven Episode wieder einzusetzen. Es wird empfohlen, mit der niedrigsten möglichen Tagesdosis in Monotherapie zu beginnen und insbesondere wenn die Mutter stillt - die Dosis lang- 
sam aufzutitrieren und dabei das Kind sorgfältig auf mögliche Nebenwirkungen hin zu beobachten.

SSRI. Selektive Serotonin-Wiederaufnahmehemmer (SSRI) sind in Bezug auf postpartale Depressionen bisher in Studien am besten untersucht. Sie besitzen eine gute Wirksamkeit bei einem günstigen Nebenwirkungsprofil, insbesondere auch im Hinblick auf eine mögliche oder intentionale Überdosierung, und meist ist die 1-malige Gabe am Tag ausreichend.

\section{Tipp für die Praxis}

Bei der Neueinstellung in der Postpartalzeit sollte nach Nutzen-Risiko-Abwägung ein Präparat gewählt werden, zu dem möglichst viele Informationen zur Stillzeit vorliegen [18].

\section{Psychopharmakotherapie in der Stillzeit}

Die Psychopharmakotherapie einer postpartalen Depression in der Stillzeit stellt sich nach unserer klinischen Erfahrung eher unkompliziert dar, wenn dabei einige Voraussetzungen beachtet werden. Hilfreich sind dabei besonders auch die Informationen des Pharmakovigilanz- und Beratungszentrums für Embryonaltoxikologie in Berlin, wo in den vergangenen Jahren systematisch Erfahrungen zum Einsatz verschiedener Medikamente in Schwangerschaft und Stillzeit dokumentiert worden sind und über eine Datenbank (www. embryotox.de) zugänglich gemacht werden.

Die Datenbank embryotox.de informiert über Medikamente während der Stillzeit.

In Buchform sind die Profile von nahezu jedem zugelassenen Psychopharmakon und die bisherigen Erfahrungen in Schwangerschaft und Stillzeit verfügbar, was bei der individuellen Nutzen-Risiko-Abwägung für die einzelne Patientin hilfreich sein kann [18].

\section{Nutzen-Risiko-Abwägung - Antidepressiva und Stillen.} Grundsätzlich muss eine Transmission des Wirkstoffs über die Muttermilch angenommen werden, allerdings ist nicht vollständig geklärt, ob die Milch-Plasma-Ratio ausschlaggebend für das Auftreten von Nebenwirkungen bei gestillten Kindern verantwortlich ist. Wenn der Verdacht auf Nebenwirkungen beim Kind (wie Irritabilität, Sedierung, unzureichende Gewichtszunahme, Veränderung des Schlaf- oder Trinkrhythmus) besteht, kann zunächst die Dosis der Medikation reduziert, alternativ auch die Ernährung des Kindes auf zusätzliche
Flaschennahrung umgestellt werden. Ob sich eine Patientin bei guter Wirksamkeit eines Medikaments auf ihre depressive Symptomatik bei Auftreten von Nebenwirkungen beim Kind für eine Umstellung der Medikation oder für das Abstillen entscheidet, muss erneut individuell mit der Patientin und ggf. dem Pädiater im Rahmen einer Nutzen-Risiko-Abwägung erörtert werden. Bei Frühgeborenen sollte eine individuelle Beurteilung mit dem Neonatologen/Pädiater erfolgen, wenn die Mutter medikamentös antidepressiv behandelt wird. Im Laufe der letzten Jahre hat sich nach unserer Erfahrung zunehmend gezeigt, dass bei den meisten Präparaten auch hier die Vorteile des Stillens überwiegen [18].

Psychotherapie. In der Akutsituation ist supportive Psychotherapie wirksam und sinnvoll. Diese orientiert sich zunächst sehr konkret an der Situation der Mutter in ihrem sozialen Gefüge. Nach einer Entaktualisierung der Symptomatik zeigt sich in der Regel dann, ob eine psychotherapeutische Weiterbetreuung sinnvoll ist, z.B., wenn Persönlichkeitsmerkmale wie eine ausgeprägte Selbstwertproblematik oder innerfamiliäre Konflikte zutage treten. Eine gute Wirksamkeit zeigte sich für interpersonelle Psychotherapie, für die es ein spezielles Modul zur postpartalen Depression gibt („Rollenwechsel“ [11,26]), und kognitive Verhaltenstherapie.

Psychotherapie ist in der Akutsituation sinnvoll und wirksam.

Insgesamt zeigen psychotherapeutische Therapieverfahren eher mittlere bis mäßige, medikamentöse Therapieverfahren höher ausgeprägte Effektstärken $[11,24]$, alle Studien dazu unterliegen allerdings deutlichen Limitationen (kleine Fallzahlen, Fehlen von Kontrollgruppen, kurze Beobachtungsdauer).

\section{Andere nicht medikamentöse Therapieverfahren.}

In Einzelstudien fanden sich positive Effekte der Lichttherapie und anderer alternativer Verfahren. Jedoch besteht insgesamt zu Omega-3-Fettsäuren, Akupunktur, Massage, Hypnose, Lichttherapie oder Schlafentzug bisher keine ausreichende empirische Evidenz [13,25]. Ebenso liegt für die Empfehlung einer Hormontherapie zur Depressionsbehandlung in der Postpartalzeit (Östrogen, Progesteron) gegenwärtig keine Evidenz vor. Zumindest bei therapieresistenten postpartalen Depressionen (die nach unseren Erfahrungen selten vorkommen) soll auch die additive Gabe von Östrogenen sinnvoll sein [25]. Die in den 1960er-Jahren propagierte Gabe von Progesteron als Prophylaxe für 
Die individuelle Haltung der Patientin zum Stillen sollte in die Entscheidungsfindung einbezogen werden. Manche Patientinnen wollen aus Sicherheitsgründen abstillen, um jegliche Nebenwirkungen beim Kind zu vermeiden, was die postpartale Medikation erleichtert. Für andere Frauen ist das Thema Stillen sehr wichtig, sodass die generelle Empfehlung zum Abstillen oft zu mangelndem Vertrauen in der therapeutischen Beziehung und zu medikamentöser Non-Compliance führt. Gerade depressive Mütter erleben es häufig als eine zusätzliche Belastung, nicht stillen zu „dürfen“ und rutschen noch weiter in eine depressive Spirale („Ich bin ja sowieso eine schlechte Mutter. Stillen ist doch das einzig Gute, was ich für mein Kind tun kann.
Und das darf ich jetzt auch nicht mehr."). Besser ist es, eine detaillierte Nutzen-RisikoAbwägung mit der betroffenen Frau und ggf. auch ihrem Partner im Hinblick auf eine antidepressive Therapie durchzuführen und die Frau in ihrer Entscheidungsfindung zu unterstützen.

Falls die Entscheidung zum Abstillen fällt, muss überlegt werden, ob dies medikamentös erfolgen sollte oder nicht. Üblicherweise wird zum primären Abstillen direkt nach der Entbindung eine Tablette Cabergolin eingenommen, um den Milcheinschuss nach Möglichkeit zu verhindern. Prolaktinhemmer wie Cabergolin und das früher verwendete Bromocriptin, das inzwischen wegen der starken Nebenwirkungen als obsolet gilt (RoteHand-Brief), sind potenziell psychisch de- stabilisierend und damit psychose- und depressionsfördernd. Insbesondere psychisch vorerkrankten Patientinnen mit hohem Rezidivrisiko (z. B. bei bipolarer Störung) möchte man jedweden zusätzlichen Faktor ersparen, der zur Remanifestation der Erkrankung führen könnte. Zum anderen muss aber bedacht werden, dass ein konservatives Abstillen mit kühlenden Umschlägen, straffem $\mathrm{BH}$, speziellen Tees und homöopathischen Mitteln langwierig und mit Stress verbunden ist. Gerade wenn vonseiten der Patientin Ambivalenz zum Thema Stillen besteht und die Entscheidung zum Abstillen fällt, ist der Einsatz einer Tablette Cabergolin dann die weit weniger belastende Lösung. postpartale Depressionen lässt sich auf dem Hintergrund der Datenlage nicht generell empfehlen, kann aber im Einzelfall hilfreich sein.

EKT. Nur bei schwerem, therapieresistentem Verlauf einer postpartalen Depression sollte nach den Leitlinien zur Therapie der Depression auch eine elektrokonvulsive Therapie (EKT) in Betracht gezogen werden - wenn keine Kontraindikationen (insbesondere im Hinblick auf die Narkose) vorliegen.

Soziale Unterstützung. Mit Einführung des Bundeskinderschutzgesetzes 2012 hat auch die Unterstützung junger Mütter und Familien im Rahmen der Prävention an Bedeutung gewonnen. Unter dem Begriff „Frühe Hilfen“ wurden lokale und regionale Unterstützungssysteme mit koordinierten Hilfsangeboten für Eltern und Kinder gebildet, die sich an Familien ab Beginn der Schwangerschaft und in den ersten Lebensjahren (Schwerpunkt Altersgruppe der 0- bis 3-Jährigen) wenden. Neben alltagspraktischer Unterstützung wollen Frühe Hilfen insbesondere einen Beitrag zur Förderung der Beziehungs- und Erziehungskompetenz von (werdenden) Müttern und Vätern leisten.

Für junge Familien stehen ab Beginn der Schwangerschaft verschiedene Hilfsangebote zur Verfügung.
Im Rahmen der postpartalen Depression hat sich dabei das Konzept der „Familienhebammen“ als besonders hilfreich erwiesen, die vom Jugendamt finanziert werden und betroffene Frauen ab der Schwangerschaft bis zum Ende des 1. Lebensjahrs des Kindes begleiten. Von postpartaler Depression betroffene Patientinnen erleben diese personelle Beratung und Unterstützung durch eine erfahrene Hebamme oft als sehr hilfreich, entlastend und nicht zuletzt auch weniger stigmatisierend als den Kontakt zu psychiatrischen Einrichtungen.

\section{Tipp für die Praxis}

Ein wichtiger Baustein in der Therapie der postpartalen Depression stellt auch die Unterstützung der Patientin in ihrer sozialen Situation dar, z. B. durch Verlängerung der Hebammenbetreuung in der Nachsorge, die Beantragung einer Familienhebamme über das Gesundheits- oder Jugendamt, die Finanzierung einer Haushaltshilfe durch die Krankenkasse, die Einbindung von Angehörigen und des Partners (z. B. durch Elternzeit/Vätermonate) und den Kontakt zu Selbsthilfegruppen (z. B. www.schatten-und-licht.de). 


\section{Management von Risikopatientinnen}

Im Vorfeld der Therapie einer postpartalen Depression stehen die sorgfältige Begleitung von Frauen mit erhöhtem Risiko und das aufmerksame Screening nach affektiven Symptomen in der Peripartalzeit. Ein erhöhtes Risiko besteht für Frauen

- mit einer rezidivierenden depressiven Störung,

- mit einer bipolaren Störung,

- mit einer Psychose in der Vorgeschichte oder

- mit einer postpartalen Depression nach einer vorherigen Schwangerschaft.

Diesen Frauen sollte bereits bei Kinderwunsch oder spätestens nach eingetretener Schwangerschaft ein konkretes Beratungsangebot gemacht werden, das eine medikamentöse Prophylaxe und das peripartale Management umfasst [18]. Häufig entwickeln die Frauen mit dem Wissen um ihr erhöhtes Risiko auch ausgeprägte Ängste vor einer erneuten Schwangerschaft.

\section{Differenzialdiagnostik der post- partalen psychischen Störungen}

\section{Postpartale Angst- und Zwangsstörungen}

Frauen mit Angst- oder Zwangserkrankungen in der Vorgeschichte haben ein erhöhtes Risiko, postpartal an einer Depression zu erkranken [14], naturgemäß kann es nach der Geburt aber auch zum Rezidiv der vorbestehenden Erkrankung kommen. Wie oben erwähnt, können Panikattacken als Teil postpartaler Depressionen auftreten (Paniktyp) und klingen in der Regel unter antidepressiver Behandlung wieder ab. Auch der Beginn einer typischen Panikstörung nach der Entbindung oder die Exazerbation einer vorbestehenden Angst- und insbesondere Panikstörung kann vorkommen $[6,27]$.

Neben Zwangsgedanken als Charakteristikum einer bestimmten Subform der postpartalen Depression (Zwangstyp) tritt eine Zwangsstörung selten auch erstmalig nach der Geburt auf, am ehesten bei Frauen mit Zwangssymptomatik in der Vorgeschichte.

\section{Fallbeispiel 3: Schwangerschaft unter Medikation [18]}

Nach postpartaler Depression mit Zwangsgedanken 2. Schwangerschaft unter Antidepressiva

Frau Y., 32 Jahre alt, stellt sich in der 23. SSW ihrer 2. Schwangerschaft zur Beratung vor. Bereits seit ihrer Jugend leidet sie an einer rezidivierenden depressiven Störung und wird antidepressiv behandelt, hatte aber auf Anraten ihrer Psychiaterin in der 1. Schwangerschaft alle Medikamente abgesetzt. Da sie nach ihren Angaben „psychisch fast dekompensiert“ ist, hatte sie sich an eine andere Ärztin gewandt, die ihr zu einem Therapieversuch mit Nortriptylin geraten hatte, das 4 Wochen vor Entbindung wieder abgesetzt wurde. Drei Wochen postpartal hatte die Patientin eine depressive Symptomatik mit schweren Zwangsgedanken entwickelt, die unter Paroxetin und Amitriptylin erst langsam wieder remittierte. In der aktuellen Schwangerschaft ist Frau Y. zum Zeitpunkt der Vorstellung bei uns unter $25-50 \mathrm{mg} / \mathrm{d}$ subdepressiv, sodass die Empfehlung zur Erhöhung auf $75 \mathrm{mg} / \mathrm{d}$ erfolgt. Darunter wird die Patientin euthym und fühlt sich subjektiv viel besser, bewältigt Alltag und Beruf, insbesondere hat sich auch der Schlaf verbessert. Die behandelnde Psychiaterin hatte ihr allerdings davon abgeraten und auch die von uns eingeholte Expertise des Pharmakovigilanz- und Beratungszentrums für Embryonaltoxikologie in Zweifel gezogen. Von unserer Seite erfolgt die Empfehlung, ca. 10 Tage vor dem errechneten Entbindungstermin Amitriptylin auf $25 \mathrm{mg} / \mathrm{d}$ zu reduzieren. Mit der Patientin wird im Rahmen eines peripartalen Managementkonzepts [18] ein Geburtsplan erstellt, der auch festlegt, dass direkt nach der Entbindung die Dosis wieder auf $75 \mathrm{mg} / \mathrm{d}$ erhöht wird. Nach der Geburt eines gesunden Jungen erlebt die Patientin eine unkomplizierte Postpartalzeit ohne Rezidiv ihrer Erkrankung.

\section{Bewertung}

Die Patientin selbst hatte eine schlechte Erfahrung mit ihrer 1 . Schwangerschaft ohne Medikation gemacht und war durch die Haltung ihrer Psychiaterin noch zunehmend verunsichert. Entlastend für sie war in der Beratung, dass sie nicht um jeden Preis ohne Medikamente auskommen müsse, bloß, weil sie schwanger sei und auch stillen wolle. Bereits eine leichte Dosiserhöhung - nach dem Prinzip „so wenig wie möglich, aber so viel wie nötig“ - besserte die psychische Situation der Patientin und damit auch ihre Lebensqualität in der Zeit der Schwangerschaft (weiterführende Informationen dazu bei [18]). 


\section{Postpartale Belastungsstörungen}

Bis zu einem Drittel der Frauen erlebt die Geburt ihres Kindes als traumatisches Ereignis [28], manchmal fallen betroffene Frauen bereits unter der Geburt mit dissoziativen Zuständen auf. Dieses subjektive Erleben, das nicht immer mit dem objektiven Geburtsverlauf übereinstimmen muss, kann zu Anpassungsstörungen und sogar zum Vollbild der posttraumatischen Belastungsstörung (PTBS) führen, die unbehandelt einen hohen Chronifizierungsgrad aufweist. Mit einer Prävalenz von 1,3-2\% posttraumatischer Belastungsstörungen bei Anwendung von DSM-5-/ICD-10-Kriterien [29] handelt es sich dabei um ein relativ häufiges Phänomen, das in Geburtshilfe und Psychiatrie bisher noch kaum wahrgenommen wird.

Bei bis zu einem Drittel der Frauen wird die Geburt als traumatisches Erlebnis empfunden.

\section{Tipp für die Praxis}

Ob es sich bei einer Entbindung um ein Trauma im engeren Sinne handeln kann, wird immer wieder kontrovers diskutiert, auch wenn die aktuellen DSM-Kriterien der PTBS nicht mehr ein Trauma „katastrophalen Ausmaßes“ verlangen und das subjektive Erleben bei Traumatisierung zunehmend an Bedeutung gewinnt [30].

Risikofaktoren für ein traumatisches Erleben der Geburtssituation sind u.a.

- sekundäre Sectio

- Geburtskomplikationen wie Präeklampsie oder HELLP-Syndrom

- traumatische Vorerfahrungen

- eine Persönlichkeitsdisposition mit hoher Kontrollbedürftigkeit

Betroffene Frauen beschreiben meist Gefühle von Hilflosigkeit, Ausgeliefertsein und verletzten Schamgefühlen, die Umwelt wurde in der Geburtssituation als unsensibel und rücksichtslos erlebt [16,30].

Traumatische Vorerfahrung. Nicht selten werden in der Vorgeschichte der Frau liegende Traumata (z. B. Missbrauchs- und Gewalterfahrungen) durch Schwangerschaft und Geburt reaktualisiert, bei Fällen von sexuellen Missbrauchserfahrungen oft bereits in der Schwangerschaft oder als Dissoziation unter der Geburt [31]. Traumatische Vorerfahrungen stellen einen Risikofaktor für die Entwicklung einer PTBS nach der Ent- bindung dar, die Prävalenz liegt in dieser Gruppe sogar bei $6-8 \%[32]$.

Erinnerungen an die Geburtssituation. Das Vorhandensein sehr lebhafter, oft auch negativer Erinnerungen an die Geburtssituation, wie sie von vielen Frauen nach der Entbindung erlebt werden, ist dabei alleine noch nicht pathologisch. Bei postpartalen Belastungsstörungen kommen Intrusionen und Flashbacks hinzu, häufig ausgelöst durch Assoziationen an die Geburtssituation. Vor „dem inneren Auge“ laufen die Geburt oder die Begleitumstände immer wieder „wie ein Film“ ab. Begleitend kommt es häufig zu Reizbarkeit, innerer Gefühllosigkeit (emotionaler Taubheit), vegetativen Symptomen und starken Angstzuständen.

PTBS führen nicht selten zu Störungen in der Mutter-Kind-Beziehung und in der Partnerschaft.

\section{Tipp für die Praxis}

Die hohe Komorbidität mit depressiver Symptomatik [32] führt nicht selten dazu, dass zwar die Diagnose einer postpartalen Depression gestellt, die Symptome der Belastungsstörung aber übersehen werden.

Therapie. Nach unserer klinischen Erfahrung sind posttraumatische Belastungsstörungen nach der Entbindung in der Regel gut behandelbar - insbesondere wenn es gelingt, Geburtshelfer und Hebammen in den therapeutischen Prozess miteinzubeziehen. Betroffene Frauen erleben oft die Nachbesprechung („Postpartum Talk“) und Klärung bestimmter Abläufe mit den an der Geburt Beteiligten als entlastend [33]. Von einem strukturierten Debriefing wird abgeraten. Handelt es sich um eine ausgeprägte Symptomatik und insbesondere bei deutlichen depressiven Symptomen, ist der Einsatz von serotonerg wirksamen Antidepressiva (z.B. SSRI) anzuraten.

Folgeschwangerschaft. Nicht selten werden aufgrund eines traumatischen Geburtserlebens weitere Schwangerschaften vermieden. Wird die Patientin trotz dieses Vermeidungsverhaltens erneut schwanger, kommt es oft zur Reaktualisierung der früheren Symptomatik in der Schwangerschaft, verbunden mit starken Geburtsängsten. „Wie ein Film“ kann das ganze Geschehen wieder präsent werden. Die betroffenen Frauen haben einen deutlich erhöhten Betreuungsbedarf in der Schwangerschaft sowohl durch den Gynäkologen/ Geburtshelfer als auch durch die Hebammen. Manchen hilft der Kontakt zu einer Beleghebamme, weil durch den kontinuierlichen Kontakt Ängste reduziert und 
Autonomie gestärkt werden. Auch die Frage nach einer primären Sectio wird nicht selten gestellt, die durchaus indiziert sein kann. Allerdings ist es für manche Patientinnen auch hilfreich, wenn eine spontane Entbindung „unter anderen Vorzeichen“ als positives Erlebnis verbucht werden kann.

\section{Tipp für die Praxis}

Die typischen Symptome einer posttraumatischen Belastungsstörung können von einer deutlich depressiven Symptomatik begleitet werden. Frauen mit depressiver Symptomatik nach der Entbindung sollten daher auch immer aufmerksam auf Intrusionen und Flashbacks sowie Derealisationserleben exploriert werden, um mögliche Zusammenhänge mit einem traumatischen Geburtserleben nicht zu übersehen.

\section{Postpartale Psychosen}

Mit einer Häufigkeit von 1-2/1000 Geburten sind postpartale Psychosen ein eher seltenes Phänomen (0,1-0,2\% [27]). Postpartal auftretende psychotische Episoden gehören aber aufgrund ihrer schweren Symptomatik und des langwierigen Verlaufs in einer für Mutter und Baby besonderen Lebensphase zu den schwersten Störungsbildern der Psychiatrie [34] und machen in der Regel die stationär psychiatrische Versorgung der Mutter - nicht selten über Wochen und Monate - unumgänglich.

Risikofaktoren. Obwohl man auch bei den postpartalen Psychosen von einer multifaktoriellen Genese ausgeht, dominieren hier - anders als bei der Depression - biologische Faktoren. Die Auftretenswahrscheinlichkeit ist über die Zeit und alle Kulturen hinweg weitgehend stabil. Am häufigsten kommen postpartale Psychosen bei Erstgebärenden vor, was den Einfluss des Life Events Geburt und Mutterschaft im Rahmen einer multifaktoriellen Genese der Erkrankung darstellt [27]. Risikofaktoren für eine postpartale Psychose sind eine vorbestehende bipolare Störung und eine positive Familienanamnese für affektive Störungen.

Bei bipolar erkrankten Frauen besteht ein $20 \%$ iges Risiko für eine psychotische und ein $50 \%$ iges Risiko für jede Form einer affektiven Episode post partum [35].

Beginn. Während sich postpartale Depressionen schleichend über Wochen und Monate entwickeln, beginnen postpartale Psychosen zu 75\% innerhalb der ersten 2 Wochen nach der Entbindung [1,27]. Bei 50\% der Episoden traten erste Symptome bereits zwischen dem 1. und 3. postpartalen Tag auf [34]. Schwangeren mit einem Risiko für postpartale Psychosen muss daher besondere Aufmerksamkeit zukommen (weiterführende Informationen s. [18]).

Alle Arten von Psychosen und damit auch alle Arten von Symptomen können nach einer Entbindung auftreten, besonders häufig treten auf:

- bipolare affektive (manische) oder schizoaffektive (schizomanische) Symptome oder

- akute polymorphe Psychosen mit ausgeprägter Antriebssteigerung, Erregung und desorganisiertem Verhalten $[8,27,34]$.

Thematisch sind die Inhalte in der Regel auf das Kind und die Geburt fokussiert (wie etwa der Größenwahn, das Jesuskind geboren zu haben), was auch als pathoplastischer Effekt der Geburt bezeichnet wird [8].

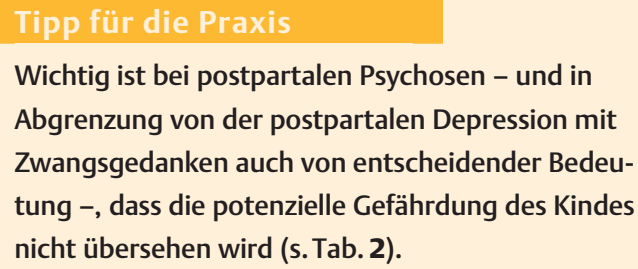

Therapie der postpartalen Psychose. Die Therapie richtet sich bei postpartalen Psychosen nach dem klinischen Bild. Da es sich in der Regel um sehr akute Zustände mit rapider Verschlechterung innerhalb von Tagen oder sogar Stunden handeln kann [34], sollte die Therapie einer postpartal auftretenden manischen oder psychotischen Symptomatik unmittelbar erfolgen und immer durch einen stationären psychiatrischen Aufenthalt eingeleitet werden. Die Verantwortung für die Betroffene kann keinesfalls von den Angehörigen übernommen werden, in Einzelfällen wird auch eine zwangsweise Unterbringung notwendig. Kann das Fortsetzen des Stillens trotz der psychotischen Symptomatik in Erwägung gezogen werden, sollte die Auswahl der Medikation nach sorgfältiger Nutzen-RisikoAbwägung erfolgen.

\section{Tipp für die Praxis}

Nach Remission der akuten Phase sollte aufgrund der Rezidivneigung (50\%iges Risiko im Falle einer erneuten Entbindung [36]) ein besonderer Schwerpunkt auf die Prophylaxe gelegt werden. 


\section{Postpartale psychische Störungen und männliches Geschlecht}

Die wenigen zu dem Thema vorhandenen Untersuchungen zeigen, dass auch Männer nach der Geburt eines Kindes gefährdet sind, Depressionen, Angststörungen oder Psychosen zu entwickeln, insbesondere bei vorbestehender Erkrankung. Der Geburt wird dabei die Rolle eines relevanten Lebensereignisses zugeschrieben, das einen Baustein in der multifaktoriellen Genese der Erkrankungen darstellt.

Die EPDS ist auch als Screening-Instrument für Männer validiert und liegt in einer deutschen Fassung vor [8].

In der Literatur findet sich eine Inzidenz der paternalen postnatalen Depression zwischen 1,2 und 25,5\% in der Allgemeinbevölkerung; bei Männern, deren Frauen an einer postpartalen Depression litten, erhöhte sich die Inzidenz auf 24-50\% [37]. Ebenso wie bei den Müttern ist in den eingetretenen Lebensveränderungen und den notwendigen Anpassungsvorgängen mit dem Übergang von der Dyade zur Triade ein wesentlicher ursächlicher Faktor für paternale postnatale depressive Verstimmungen zu sehen, wobei sich für den Vater noch die zusätzliche Herausforderung stellt, seinen Platz neben der oft sehr engen Beziehung zwischen Mutter und Kind zu finden, aus der er sich schnell ausgeschlossen fühlen kann.

Der enge Zusammenhang zwischen postpartalen Depressionen mit Ängsten und Insuffizienzgefühlen wird auch bei der paternalen postnatalen Depression deutlich [8].

Vorbestehende psychische Erkrankungen wie Angstoder Zwangsstörungen, aber auch die Exazerbation einer psychotischen Störung können bei Männern in Folge der Geburt eines Kindes auftreten bzw. sich ver- schlechtern. Systematische Untersuchungen mit Fokus auf dem Einfluss solcher familiären Veränderungen sind allerdings bisher nicht vorhanden, lediglich in kleinen Fallserien wird darüber berichtet.

Dass auch die Väter der Kinder die Umstände der Schwangerschaft und Geburt und besonders das Auftreten von Komplikationen als traumatisch erleben können, ist aus unserer eigenen klinischen Arbeit gut bekannt. In Kasuistiken und Untersuchungen konnte gezeigt werden, dass die Anwesenheit des Mannes bei der Entbindung einen wesentlichen Prädiktor für die Entwicklung posttraumatischer Symptome darstellt. In einer eigenen Studie zu Geburtserleben und postnataler Befindlichkeit bei über 400 Männern und Frauen konnte der Einfluss der Geburtserfahrung auf das Geburtserleben dargestellt werden [38]. Väter, bei denen postpartal ein erhöhter Depressivitätsscore in der Edinburgh Postnatal Depression Scale (EPDS) auffiel, zeigten ein schlechteres emotionales Geburtserleben als die Gesamtgruppe. Signifikant häufiger trat Depressivität bei denjenigen Männern auf, deren Erwartungen an die Geburt nicht erfüllt wurden. Klinisch bedeutsam ist dies insbesondere vor dem Hintergrund, dass Depressivität des Vaters in der Postpartalzeit auch unabhängig vom Auftreten einer postpartalen Depression bei der Mutter - ein Prädiktor für die Entwicklung psychischer Störungen bei den betroffenen Kindern ist [39].

\section{Fazit für die Praxis}

Auch Männer können postpartal psychische Probleme entwickeln und zwar in erster Linie depressive Störungen. Dabei gibt es eine hohe Korrelation zwischen Depressionen der Mutter und der Auftretenswahrscheinlichkeit beim Vater sowie klare Hinweise auf potenzielle Auswirkungen beim Kind, weshalb auch eine väterliche Depression erkannt und behandelt werden sollte. 
- Postpartale Depressionen sind mit 10-15\% die häufigste psychische Störung in der Postpartalzeit. Jährlich sind in Deutschland 70000 Mütter, ihre Kinder und Partner betroffen.

- Postpartale Depressionen entwickeln sich in der Regel schleichend in den ersten Wochen und Monaten nach der Geburt eines Kindes. Differenzialdiagnostisch sind sie von postpartalen Angst- und Zwangsstörungen, Belastungsstörungen und postpartalen Psychosen abzugrenzen.

- Postpartale Depressionen sind leicht zu diagnostizieren; erste Hinweise ergeben sich bei Anwendung des ScreeningInstruments Edinburgh Postnatal Depression Scale (EPDS), das auch für den Einsatz in der gynäkologischen Praxis geeignet ist.

- Eine leitliniengerechte Behandlung der postpartalen Depression richtet sich nach der im Vordergrund stehenden Symptomatik und folgt den üblichen Kriterien der antidepressiven Therapie: medikamentös, psychotherapeutisch und soziotherapeutisch - allerdings bei einer stillenden Mutter unter entsprechender Nutzen-Risiko-Abwägung hinsichtlich der Antidepressivagabe.

- Ein wichtiger Therapiebaustein bei psychischen Störungen in der Postpartalzeit ist die soziale Unterstützung der Frau, z. B. durch verlängerte Hebammenbetreuung, Haushaltshilfe durch die Krankenkasse, Familienhebamme, Einbindung des Partners (Elternzeit) und Selbsthilfegruppen.

- Da auch Väter postpartal depressiv sein können, empfiehlt sich auch diesbezüglich eine verstärkte Aufmerksamkeit. Depressivität bei der Mutter erhöht die Wahrscheinlichkeit depressiver Symptome beim Vater.
- Da sowohl mütterliche als auch väterliche Depressionen Auswirkungen auf die kindliche Entwicklung haben können, sollte die suffiziente Behandlung der postpartalen Depression so früh wie möglich erfolgen.

- Ein nicht unerheblicher Teil an Frauen erlebt die Geburt ihres Kindes traumatisch. Risikofaktoren für die Entwicklung einer posttraumatischen Belastungsstörung (PTBS) nach einer Entbindung sind Gewaltund Missbrauchserfahrungen, die in der Schwangerschaft reaktualisiert oder unter der Geburt als Dissoziation auftreten können.

- Wegen der potenziellen Gefährdung des Kindes muss bei psychotischen Symptomen (z. B. Wahnsymptome oder akustische Halluzinationen) eine stationäre Behandlung der Mutter herbeigeführt werden.

\section{Über die Autorinnen}

\section{Valenka M. Dorsch}

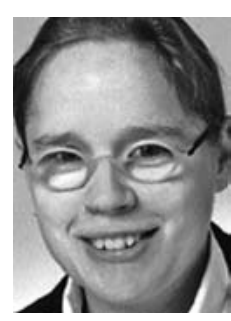

Dr. med. Jahrgang 1973. Fachärztin für Psychiatrie und Psychotherapie. Studium der Medizin in Bonn. 2006-2010 wissenschaftliche Mitarbeit in der Gynäkologischen Psychosomatik am Zentrum für Frauenheilkunde und Geburtshilfe des Universitätsklinikums Bonn. Seit 2015 LVR-Klinik Köln, Sprechstunde für psychisch kranke Frauen in Schwangerschaft und Stillzeit. Forschungsschwerpunkte: geschlechtssensible Psychiatrie, Psychopharmakotherapie in Schwangerschaft und Postpartalzeit, Neonatizid, Männer und Peripartalzeit.

\section{Anke Rohde}

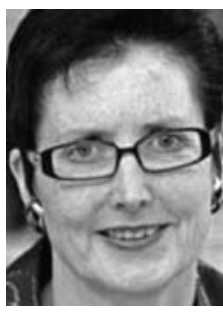

Prof. Dr. med. Jahrgang 1954. Fachärztin für Psychiatrie, Psychotherapie und Nervenheilkunde. 1997-2015 Professorin für Gynäkologische Psychosomatik und Leiterin des entsprechenden Funktionsbereichs am Zentrum für Frauenheilkunde und Geburtshilfe des Universitätsklinikums Bonn. Forschungs- und Arbeitsschwerpunkte: psychische Störungen und Psychopharmakotherapie in der Schwangerschaft und Postpartalzeit, peripartales Management, psychische Belastungen in der Pränatalmedizin, Hormone und Psyche, Infantizid/Neonatizid.

\section{Korrespondenzadresse}

Dr. med. Valenka M. Dorsch

LVR-Klinik Köln

Sprechstunde für psychisch kranke Frauen in Schwanger-

schaft und Stillzeit

Wilhelm-Griesinger-Straße 23

51109 Köln

E-Mail: valenka.dorsch@lvr.de 


\section{Interessenkonflikt}

Die Autoren erklären, dass kein Interessenkonflikt in Bezug auf das Manuskript besteht.

\section{Literatur}

1 Sit DK, Wisner KL. Identification of postpartum depression. Clin Obstet Gynecol 2009; 52: 456-468

2 Kendell RE, Chalmers JC, Platz C. Epidemiology of puerperal psychoses. Br J Psychiatry 1987; 150: 662-673; Erratum in: $\mathrm{Br}$ J Psychiatry 1987; 151: 135

3 Valdimarsdóttir U, Hultman CM, Harlow B et al. Psychotic illness in first-time mothers with no previous psychiatric hospitalizations: a population-based study. PLoS Med 2009; 6: e13

4 Jones I, Chandra PS, Dazzan P et al. Bipolar disorder, affective psychosis, and schizophrenia in pregnancy and the post-partum period. Lancet 2014; 384: 1789-1799

5 Halligan SL, Murray L, Martins C et al. Maternal depression and psychiatric outcomes in adolescent offspring: a 13-year longitudinal study. J Affect Disord 2007; 97: 145-154

6 Howard LM, Molyneaux E, Dennis CL et al. Non-psychotic mental disorders in the perinatal period. Lancet 2014; 384: 1775-1788

7 Riecher-Rössler A, Rohde A. Diagnostic Classification of perinatal Mood Disorders. In: Riecher-Rössler A, Steiner M, eds. Perinatal Stress, Mood and Anxiety Disorders. From Bench to Bedside. Basel, Freiburg, Paris: Karger; 2005: 6-27

8 Rohde A, Marneros A, Hrsg. Geschlechtsspezifische Psychiatrie und Psychotherapie. Stuttgart: Kohlhammer; 2007

9 Bloch M, Rotenberg N, Koren D et al. Risk factors associated with the development of postpartum mood disorders. J Affect Disord 2005; 88: 9-18

10 O’Hara MW, McCabe JE. Postpartum depression: current status and future directions. Annu Rev Clin Psychol 2013; 9: 379-407

11 Werner A, Miller M, Osborne LM et al. Preventing postpartum depression: review and recommendations. Arch Womens Ment Health 2015; 18: 41-60

12 Halbreich U, Karkun S. Cross-cultural and social diversity of prevalence of postpartum depression and depressive symptoms. J Affect Disord 2006; 91: 97-111

13 Dennis CL, Dowswell T. Interventions (other than pharmacological, psychosocial or psychological) for treating antenatal depression. Cochrane Database Syst Rev 2013; (7): CD006795

14 Milgrom J, Gemmill AW, Bilszta JL et al. Antenatal risk factors for postnatal depression: A large prospective study. J Affect Disord 2007; 108: 147-157

15 Brockington I. Motherhood and mental Health. In: Brockington I. ed. Motherhood and mental health. Oxford: Oxford University Press; 1996

16 Rohde A, Dorn A. Gynäkologische Psychosomatik und Gynäkopsychiatrie. Das Lehrbuch. Stuttgart: Schattauer; 2007

17 Rohde A. Psychiatrische Erkrankungen in der Schwangerschaft und im Wochenbett. Gynäkologe 2001; 34: 315-323

18 Rohde A, Dorsch V, Schäfer C. Psychopharmakotherapie in Schwangerschaft und Stillzeit. Behandlungsprinzipien, Leitlinien, peripartales Management. Stuttgart: Thieme; 2015b
19 Spinelli MG. Postpartum psychosis: detection of risk and management. Am J Psychiatry 2009; 166: 405-408

20 Cox J, Holden M, Sagovsky R. Detection of postnatal depression. Development of the 10-item Edinburgh Postnatal Depression Scale. Br J Psychiatry 1987; 150: 782-786

21 Bergant A, Nguyen T, Heim K et al. Deutschsprachige Fassung und Validierung der „Edinburgh postnatal depression scale“. Dtsch Med Wochenschr 1998; 123: 35-40

22 Reulbach U, Bleich S, Knörr J et al. Prä-, peri- und postpartale Depressionen. Fortschr Neurol Psychiatr 2009; 77: 708-713

23 Whooley MA, Avins AL, Miranda J et al. Case-finding instruments for depression. Two questions are as good as many. J Gen Intern Med 1997; 12: 439-445

24 Pearlstein T, Howard M, Salisbury A et al. Postpartum depression. Am J Obstet Gynecol 2009; 200: 357-364

25 DGPPN, BÄK, KBV, AWMF, AkdÄ, BPtK, BApK, DAGSHG, DEGAM, DGPM, DGPs, DGRW, Hrsg. S3-Leitlinie/Nationale VersorgungsLeitlinie Unipolare Depression - Langfassung. 2. Aufl. 2015. Im Internet: http://www.depression.versorgungsleitlinien.de; Stand: 31.07.2016

26 O'Hara MW, Stuart S, Gorman LL et al. Efficacy of interpersonal psychotherapy for postpartum depression. Arch Gen Psychiatry 2000; 57: 1039-1045

27 Brockington I. Postpartum psychiatric disorders. Lancet 2004; 363: $303-310$

28 Maggioni C, Margola D, Filippi F. PTSD, risk factors, and expectations among women having a baby: a two-wave longitudinal study. J Psychosom Obstet Gynaecol 2006; 27: 81 -90

29 Soderquist J, Wijma B, Thorbert G et al. Risk factors in pregnancy for post-traumatic stress and depression after childbirth. $\mathrm{Br}$ J Obstet Gynaecol 2009; 116: 672-680

30 Garthus-Niegel S, von Soest T, Vollrath ME et al. The impact of subjective birth experiences on post-traumatic stress symptoms: a longitudinal study. Arch Womens Ment Health 2013; 16: 1-10

31 Olde E, van der Hart O, Kleber RJ et al. Peritraumatic dissociation and emotions as predictors of PTSD symptoms following childbirth. J Trauma Dissociation 2005; 6: 125-142

32 Seng JS, Rauch SA, Resnick H et al. Exploring posttraumatic stress disorder symptom profile among pregnant women. J Psychosom Obstet Gynaecol 2010; 31: 176-187

33 Olin RM, Faxelid E. Parents' needs to talk about their experiences of childbirth. Scand J Caring Sci 2003; 17: 153-159

34 Jones I, Chandra PS, Dazzan P et al. Bipolar disorder, affective psychosis, and schizophrenia in pregnancy and the post-partum period. Lancet 2014; 384: 1789-1799

35 Di Florio A, Forty L, Gordon-Smith Ket al. Perinatal episodes across the mood disorder spectrum. JAMA Psychiatry 2013; 70: 168-175

36 Blackmore ER, Rubinow D, O'Connor TG et al. Reproductive outcomes and risk of subsequent illness in women diagnosed with postpartum psychosis. Bipolar Disord 2013; 15: 394-404

37 Paulson JF, Bazemore SD. Prenatal and postpartum depression in fathers and its association with maternal depression: a metaanalysis. J Am Med Assoc 2010; 303: 1961-1969

38 Dorsch VM, Hueneburg A, Prestien A et al. Vater sein dagegen sehr... In: Maier B, Braun C, Schuster D, Hrsg. Ver-Bindung. Frankfurt/Main: Mabuse-Verlag; 2010

39 Ramchandani P, Stein A, O'Connor T et al. Depression in men in the postnatal period and later child psychopathology: a population cohort study. J Am Acad Child Adolesc Psychiatry 2008; 47 $390-398$ 


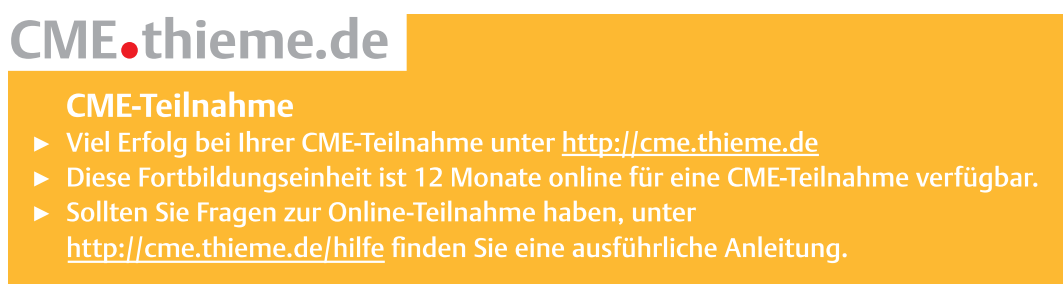

\section{CME-Fragen}

Welche Aussage zu postpartalen psychischen Störungen trifft zu? Postpartale psychische Störungen ...

A sind nicht krankheitswertig, sondern stellen normale Anpassungsvorgänge dar.

B wurden erstmals von Hippokrates im 5. Jh. v. Chr. beschrieben.

C betreffen nur Erstgebärende.

D erklären sich allein durch die Hormonumstellung nach der Entbindung.

E betreffen ausschließlich Frauen.

2 Welche Aussage trifft nicht zu? „Babyblues“...

A bezeichnet postpartale Stimmungsschwankungen mit raschem Wechsel zwischen Euphorie und Weinerlichkeit/Reizbarkeit/ Insuffizienzgefühlen.

B betrifft ca. 15-17\% der Frauen nach der Entbindung.

C beginnt in der Regel um den 3. -5. Tag nach der Geburt.

D gilt bei besonders stark ausgeprägten Symptomen als Vulnerabilitätsmerkmal zur Entwicklung einer postpartalen Depression.

E erfordert aufgrund des selbstlimitierenden Verlaufs in der Regel keine spezifische Therapie.

3 Welche Aussage trifft zu? Postpartale Depressionen ...

A sind als „Wochenbettdepression“ in der ICD-10 codierbar.

B unterscheiden sich in ihrer syndromalen Charakterisierung von depressiven Episoden zu anderen Zeiten im Leben einer Frau.

C führen trotz mangelndem Verständnis für die Bedürfnisse des Säuglings nur selten zu einer gestörten Mutter-Kind-Bindung.

D sind pathophysiologisch durch die konstant nachweisbaren Unterschiede bei den Spiegeln gonadaler Steroide im Plasma von Frauen in der Schwangerschaft bzw. im Wochenbett zu erklären.

E weisen thematisch oft einen Bezug zur Mutterschaft auf (z. B. Insuffizienzgefühl als Mutter).

4 Welche Aussage trifft zu? Postpartale Depressionen beginnen in der Regel ...

A abrupt innerhalb von 3-5 Tagen nach der Entbindung.

B abrupt innerhalb von 14 Tagen nach der Entbindung.

C schleichend innerhalb von 14 Tagen nach der Entbindung.

D schleichend innerhalb der ersten Wochen und Monate nach der Entbindung.

E erst nach der Abstillperiode, frühestens 6 Monate nach der Entbindung.

5 Welche Aussage zur EPDS (Edinburgh Postnatal Depression Scale) trifft nicht zu?

A Sie dient als Screening-Instrument auf Depressivität und Angst.

B Sie ist als Selbstbeurteilungsfragebogen konzipiert.

C Die EPDS steht in einer deutschen Fassung zur Verfügung.

D Ein niedriger EPDS-Score weist auf hohe Suizidgefahr hin.

E Sie kann auch in der gynäkologischen Praxis unkompliziert eingesetzt werden.

6 Welche Aussage zu postpartalen Zwangssymptomen ist nicht richtig?

A Zwangsgedanken der Mutter sind stark mit erweitertem Suizid korreliert; eine stationär psychiatrische Behandlung der Mutter ist unumgänglich.

B Zwangsgedanken, das neugeborene Kind zu verletzen, sind im Rahmen einer postpartalen Depression nicht selten.

C Die betroffenen Mütter leiden unter großen Schuld- und Schamgefühlen.

D Vermeidungsverhalten ist eine typische Folge; nicht selten haben die Mütter Angst, mit den Kindern alleine zu sein.

E Differenzialdiagnostisch sind psychotische Symptome (z. B. Stimmenhören) auszuschließen. 
Welche Aussage trifft nicht zu? Akute Gefährdungsmomente für das Kind bestehen in der Regel insbesondere bei ...

A verdrängter oder verleugneter Schwangerschaft.

B Babyblues mit erhöhter emotionaler und affektiver Turbulenz.

C postpartaler Depression mit Suizidgedanken.

D akustischen Halluzinationen, imperativen Stimmen.

E Wahnsymptomen.

8 Welche Aussage zu postpartalen Depressionen trifft nicht zu?

A Informationen zur leitliniengerechten Therapie peripartaler Depressionen finden sich in der Leitlinie „Unipolare Depression“ (2015) der Fachgesellschaften.

B Die individuelle Therapie richtet sich nach der Ausprägung der Symptomatik und umfasst medikamentöse, psychotherapeutische und soziotherapeutische Behandlungsansätze.

c Soziale Unterstützung (z. B. verlängerte Hebammenbetreuung, Familienhebamme durch das Jugendamt, Haushaltshilfe durch die Krankenkasse, Einbindung des Partners durch Elternzeit und Selbsthilfegruppen) kann betroffene Frauen entlasten.

D Bei der postpartalen Depression sollte immer eine Meldung an das Jugendamt zur Prüfung der Kindeswohlgefährdung und Inobhutnahme erfolgen.

E Unter Monotherapie ist Stillen für viele Medikamente bei der postpartalen Depression vertretbar.

9 Welche Aussage zu postpartalen psychischen Störungen trifft nicht zu?

A Nach epidemiologischen Angaben sind 10-15\% aller Frauen von postpartaler Depressivität betroffen.

B Das Auftreten der paternalen postnatalen Depression zeigt eine starke Korrelation mit dem Vorhandensein einer postpartalen Depression bei der Mutter.

C Die Edinburgh Postnatal Depression Scale (EPDS) und die Whooley-Fragen sind validierte Methoden des Screenings auf postpartal erhöhte Depressivität.

D Anders als die depressive Symptomatik, die sich oft schleichend über Monate hinweg entwickelt, beginnen 75\% aller postpartalen Psychosen innerhalb der ersten beiden Wochen nach der Entbindung.

E Eine postpartale Belastungsstörung (PTBS) muss bei depressiver Symptomatik der Mutter differenzialdiagnostisch nicht in Betracht gezogen werden, da eine Entbindung nicht die Kriterien eines Traumas erfüllen kann.

10 Welche Aussage zu postpartaler Depression und Stillen trifft zu?

A Transmission in die Muttermilch konnte für keine antidepressive Substanz nachgewiesen werden.

B Eine psychopharmakologische Therapie muss in der Stillzeit unbedingt vermieden werden, da anhaltende negative Auswirkungen auf gestillte Kinder in einer Vielzahl von Untersuchungen beschrieben worden sind.

C Bei der Neueinstellung in der Postpartalzeit sollte nach Nutzen-Risiko-Abwägung ein Präparat gewählt werden, zu dem möglichst viele Informationen zur Stillzeit vorliegen.

D Von einer postpartalen Depression betroffenen Frauen sollte immer zum Abstillen geraten werden, um vor dem Stress geschützt zu werden, den das Stillen mit sich bringt.

E Bei Diagnosestellung einer postpartalen Depression sollte ein medikamentös unterstütztes Abstillen mit Bromocriptin erfolgen. 